\title{
La reconfiguración del campo asistencial: el caso del plan de equidad de Uruguay*
}

\section{The new configuration of the assistance fiedd: the Uruguayan equity plan}

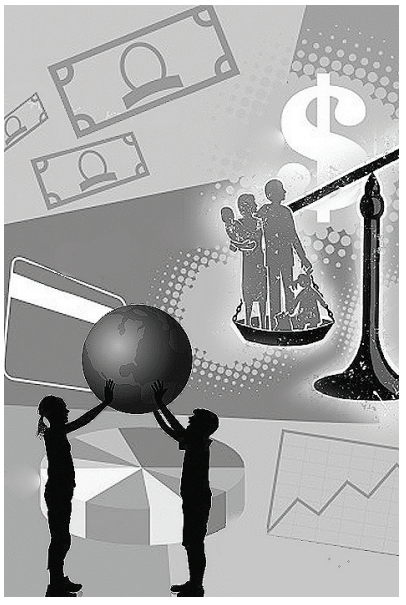

\section{Laura Vecinday**}

Resumen: La reciente y creciente consolidación de los Programas de Transferencia de Renta Condicionada (PTRC) como principal instrumento de combate a la pobreza en América Latina es el puntapié de estas reflexiones. Su puesta en práctica supone la reconfiguración del campo asistencial inaugurando o reeditando dispositivos tecnológicos e institucionales que son parte de la nueva ingeniería política de la protección social. El análisis se centró en el estudio de un caso: el Plan de Equidad - Nuevo régimen de asignaciones familiares. ${ }^{* *}$

Palabras clave: Programas de Transferencia de Renta Condicionada. Campo asistencial. Prácticas profesionales.

Abstract: The recent and increasing consolidation of the Programs for Conditioned Income Transfer (Programas de Transferencia de Renta Condicionada - PTRC) as the main instrument to fight poverty

* En el artículo se desarrollan y profundizan algunas de las reflexiones esbozadas en la ponencia presentada en el Encuentro sobre Estado, Políticas Sociales y Sociedad bajo el título "Impactos de la reingeniería política de la protección social en las prácticas profesionales. El caso del Plan de Equidad de Uruguay", Proieps, FCH, UNCPBA realizado en Tandil, Argentina en noviembre de 2012.

** Doctora en Ciencias Sociales por la Facultad Latinoamericana de Ciencias Sociales (Flacso - Sede Académica Argentina). Profesora del Departamento de Trabajo Social (Facultad de Ciencias Sociales) de la Universidad de la República en Montevideo, Uruguay. Integra el Sistema Nacional de Investigadores de la ANII. E-mail: laurave@adinet.com.uy.

*** El trabajo es producto de la investigación, aún en curso, sobre Programas de Transferencia de Renta Condicionada en América Latina y el Caribe: estudio comparado - "Bolsa Família" (Brasil), "Asignación Familiar del Plan de Equidad" (Uruguay) y "Asignación Universal por Hijo" (Argentina). Proyecto financiado por la Coordenação de Aperfeiçonamento de Pessoal no Nível Superior (Capes) de Brasil y administrado en Uruguay por la Agencia Nacional de Investigación e Innovación (ANII — Edital Mercosur Educativo). Este artículo fue elaborado en el transcurso de la estadía posdoctoral prevista en el marco del proyecto mencionado y con la orientación de la Profa. Dra. Maria Ozanira da Silva e Silva. 
in Latin America is the starting point of the following thinking. Their implementation implies a restructuring of assistance policies, launching or modifying technical and institutional devices that are part of the new political engineering of social protection. The analysis focused on a case study: the Equity Plan - a new system of family allocations.

Keywords: Programs for Conditioned Income Transfer. Assistance policies. Professional practice.

\section{Introducción}

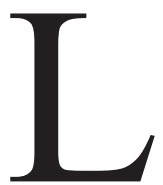

os contenidos aquí trabajados refieren al caso del Nuevo Régimen de Asignaciones Familiares del Plan de Equidad en Uruguay (AFAM-PE) implementado desde enero de 2008. El Plan de Equidad reúne un conjunto de prestaciones monetarias y no monetarias de combate a la pobreza siendo las AFAM su componente monetario.

La creación de las AFAM como prestación monetaria percibida por los trabajadores privados data de 1943 y su historia está estrechamente ligada a la evolución del sistema de seguridad social. Su población objetivo varió a lo largo del tiempo a través de modificaciones legislativas que incluyeron a nuevos grupos de trabajadores y excluyeron a otros según niveles de ingreso. Estas transferencias fueron condicionadas exigiendo la asistencia de los niños y adolescentes al sistema educativo, pero, esencialmente fueron pensadas como un complemento de los ingresos de los hogares (Plan de Equidad, s/f, p. 33).

El Plan de Equidad utiliza el instrumento de la AFAM como programa de transferencia de renta condicionada, de carácter no contributivo. Las prestaciones sociales ofrecidas por el Ministerio de Desarrollo Social (MIDES) se corresponden con distintas estrategias de combate a la pobreza. Sin embargo, en el caso de las transferencias monetarias (AFAM y pensiones a la vejez), la institución administradora es el Banco de Previsión Social (BPS) cumpliendo el MIDES un papel auxiliar, como veremos más adelante.

Midaglia y Silveira (2011, p. 243) indican que si bien las AFAM nacieron como un complemento salarial de los trabajadores formales en el ámbito de la seguridad social se convirtieron, en el actual régimen, en una estrategia de combate a la pobreza. De este modo, las contrapartidas exigidas en sus orígenes

contribuían a asegurar una sociedad "civilizada y garantista" dinámica económicamente, con mecanismos comunes y por ende universales de socialización de los 
segmentos jóvenes de población [...] se asociaban, de alguna manera, al proyecto de una nación moderna, con una mano de obra joven, educada y un mercado de empleo en expansión. Las exigencias establecidas en el nuevo régimen de Asignaciones tienden cumplir un papel distinto [...] buscan incidir en las condiciones de vida de los estratos sociales desfavorecidos.

La implementación de las AFAM-PE supuso una dualización del régimen a partir de la convivencia de prestaciones contributivas y no contributivas reuniendo, de este modo, la lógica del seguro para los trabajadores formales y la de la asistencia para poblaciones cada vez más diferenciadas. Esta dualización se explica por la definición de objetivos diferenciados de regulación poblacional en función de los grupos poblacionales a los cuales se orienta. El nuevo régimen pretende incidir en la condiciones de vida de los sectores más pobres ofreciendo un estímulo económico para la transformación de ciertos comportamientos vinculados a la educación y al cuidado de la salud. De este modo, las transferencias pretenden "lograr ciertos comportamientos por parte de los hogares, como asistencia al sistema educativo de los niños y niñas y cuidados de la salud de los niños, niñas y de las embarazadas" (Plan de Equidad, s/f, p. 33)

Las AFAM-PE son complementadas con la denominada Tarjeta Uruguay Social (TUS) cuando sus preceptores son hogares en situación de extrema pobreza. A través de la TUS se transfieren recursos monetarios para la compra de alimentos y productos de higiene. La transferencia es administrada por el MIDES adjunto al Instituto Nacional de Alimentación (INDA) y el Ministerio de Salud Pública (MSP).

Presentado brevemente el caso estudiado se propone, en primer lugar, una lectura acerca del campo asistencial en el contexto de la protección social y las principales tensiones y contradicciones que lo atraviesan.

Luego se analiza la creación de los Ministerios Sociales como la emergencia de una nueva institucionalidad que consolida a las políticas de combate a la pobreza en tanto "respuesta" a las miserables condiciones de existencia de grandes grupos de población. Este proceso se interpreta como un fuerte indicio del cambio en la orientación filosófica del sistema de protección social que desplaza el objetivo de la integración social y jerarquiza las pretensiones de inserción social.

Los apartados siguientes contienen reflexiones acerca del proceso de reconfiguración tecnológica e institucional del campo asistencial. La tecnificación creciente de los dispositivos asistenciales encuentra en los PTRC sus exponentes contemporáneos más representativos. La administración gerencial de la asistencia 
y sus demandas de conocimiento experto son dimensiones centrales para comprender el impacto de estas alteraciones en la práctica de las profesiones asistenciales.

\section{El campo asistencial y las profesiones asistenciales}

La configuración de un campo asistencial deviene de la consolidación de un esquema de protección social apoyado en dos instrumentos esenciales: los seguros sociales y la asistencia social. Los seguros sociales se configuraron como el instrumento tecnológico que posibilitó la reducción de los riesgos de la existencia, siendo la asistencia social el dispositivo destinado a los "casos sociales", los "minusválidos" y los "pobres" (Castel, 2003, p. 42). De algún modo, en la propia génesis del campo asistencial están contenidas ciertas contradicciones representadas en las tensiones entre derecho y caridad o filantropía, trabajadores y asistidos, contribuyentes y no contribuyentes etc. Así configurado, el campo asistencial siempre resultó sospechoso respecto a la amplitud y la calidad de sus prestaciones en comparación con la técnica del seguro, valorada como la posibilidad de "salir de las aporías ligadas a una visión puramente individualista de la sociedad" (Rosanvallon, 1995, p. 21). El seguro social ha sido el dispositivo más generalizado de las formas de protección típicas de los Estados de Bienestar y ha significado una forma particular de relación individuo - sociedad expresada por Rosanvallon (1995, p. 23) en los siguientes términos:

al pasar de la noción subjetiva de comportamiento y responsabilidad individual a la noción objetiva de riesgo, el seguro invitaba a mirar lo social de manera diferente [...]. El enfoque en términos de riesgo, en efecto, remite en primer lugar a una dimensión probabilística y estadística de lo social (el riesgo es calculable) que permite relegar al segundo plano el juicio sobre los individuos. Cuando las situaciones se aprehenden en términos de riesgo, la cuestión de las culpas personales y de las actitudes individuales pasa a ser secundaria. ${ }^{1}$

1. Rosanvallon (1995) atribuye la pérdida de eficacia protectora de la técnica del seguro a la emergencia de un problema de escala en relación a los riesgos. Sus argumentos, en consonancia con los análisis de los teóricos de la modernización reflexiva (Beck, Giddens, Lash, 1997), apuntan a equiparar la relevancia de los riesgos clásicos con los denominados nuevos riesgos: "la noción de riesgo sigue siendo pertinente. Pero ha cambiado de escala, como se lo subrayó con justeza. Lo que hoy en día constituye cada vez más un pro- 
La permanente búsqueda de legitimidad sociopolítica favoreció el desarrollo de acciones estatales que extendieron los beneficios del fordismo a gran parte de la sociedad a través de la asistencia médica, la vivienda y los servicios educativos. El "Estado protector" fue posible en condiciones de crecimiento de la productividad, el consumo y los salarios, y se realizó mediante la inscripción del individuo en colectivos protectores vinculados al trabajo (Castel, 2004, p. 48-51). Este crecimiento de la productividad junto a una mayor capacidad de consumo generaron las condiciones que posibilitaron esa particular configuración de la intervención pública en el campo social.

La asociación entre trabajo y protección social fortaleció la organización colectiva de los trabajadores al tiempo que promovió la mejora constante de la calidad de las prestaciones y los servicios sociales. La creciente fuerza de los movimientos y partidos obreros se apoyaba en la convicción de que cualquier mejora en sus condiciones de vida no admitía acciones individuales sino colectivas (Hobsbawm, 1995, p. 300).

Desde la década de los 1970, aproximadamente, se registran progresivas alteraciones en las formas de organización del trabajo y la producción que han contribuido a la pérdida de eficacia de un modelo bipolar de protección basado en el seguro y la asistencia. La "producción" de una gran masa de "inempleables" y la inestabilidad en las trayectorias ocupacionales son consecuencias de los procesos de reestructuración productiva que jaquearon aquel modelo bipolar. Este modelo organizó el esquema de protección social en Uruguay a lo largo del siglo $\mathrm{XX}$ en el que el seguro social supuso un conjunto de prestaciones y servicios sociales amortiguadores ante contingencias acaecidas en una relativamente estable trayectoria laboral, al tiempo que la asistencia se ha reservado históricamente para aquéllos que por diversas razones no se encuentran en condiciones de producir. $^{2}$

\footnotetext{
blema es el riesgo catastrófico: riesgos naturales (inundación, terremoto), accidentes tecnológicos mayores, daños de gran amplitud al medio ambiente. Estas amenazas ya no conciernen a individuos sino a poblaciones enteras, incluso a naciones. La socialización de los riesgos que lleva adelante el seguro ya no puede ser operativa en estos casos" (Rosanvallon, 1995, p. 29). Más que un problema de escala, Castel (2004) dirá que se trata de situaciones de peligro y no de riesgos, y que asimilar estos tipos de riesgos justifica el desmonte de las formas colectivas de protección.

2. “En esos 'treinta gloriosos' años (como dicen los franceses) que van de 1945 a 1975, siempre existió un porcentaje de personas que no encontraban empleo. Pero se trataba de un desempleo funcional y en la mayoría de los casos temporal al que la sociedad hacía frente mediante el seguro de desempleo. Para las si-
} 
Fue la generalización del trabajo en su forma asalariada el mecanismo que permitió el desarrollo de tal esquema de protección social. Su puesta en marcha suponía la existencia de una "homología de posiciones entre los distintos tipos de asalariados" (Tenti, 2000, p. 96). Cuando las trayectorias ocupacionales comienzan a caracterizarse por su creciente flexibilidad, informalidad y precariedad, aquellas protecciones vinculadas a trayectorias otrora estables, formales y sólidas hacen agua condenando a crecientes sectores de población a situaciones de desprotección y vulnerabilidad.

Para Domínguez Ugá (2004, p. 60) “o 'mundo do trabalho' sofre, assim, uma transformação. O que antes constituía uma classe social (composta por trabalhadores 'empregados' e 'desempregados') agora forma um conjunto de indivíduos atomizados: os 'competitivos' e os 'incapazes' (pobres)".

El campo asistencial es entonces reconfigurado acompañando de forma más o menos coherente las alteraciones en las formas de organización del trabajo y la producción. Surgen nuevos dispositivos de protección alejados de la lógica del seguro y situados en el campo asistencial por su carácter no contributivo. Paradójicamente, estos dispositivos se dirigen a poblaciones cuyas características no se corresponden con aquéllas que históricamente formaron parte del campo asistencial. Por ejemplo, en el caso de los PTRC, los nuevos asistidos pertenecen a familias y grupos poblacionales inempleables, es decir, "incapaces" de vender su fuerza de trabajo. ${ }^{3}$ En otras palabras, se trata de asistir a aquellos sectores de población que, estando en condiciones de trabajar, no logran integrarse al mercado laboral o su integración es tan precaria que compromete su reproducción como individuo y la de su grupo social de pertenencia.

Desde esta perspectiva, los límites del campo asistencial se han ampliado en desmedro de la expansión del seguro social abriéndose un conjunto de interrogantes acerca del signo de esta transformación en relación al bienestar de los sectores trabajadores. Si bien este análisis trasciende los objetivos del presente trabajo se advierte que no existen indicios que permitan afirmar que avanzamos hacia una

\footnotetext{
tuaciones extremas y minoritarias de exclusión social, el Estado desplegaba una estrategia asistencial de emergencia" (Tenti, 2000, p. 79).

3. Estos segmentos desprotegidos "não podem ser sumariamente identificados ao lumpem "clássico". Tais segmentos compreendem universos heterogêneos, desde aposentados com pensões miseráveis, crianças e adolescentes sem qualquer cobertura social, migrantes e refugiados, doentes estigmatizados (recordem-se os aidéticos pobres) até trabalhadores expulsos do mercado de trabalho (formal e informal)" (Netto, 2012).
} 
transformación en la lógica de los dispositivos bismarckianos (contributivos y no contributivos). Por lo tanto, toda alteración continúa respondiendo a esta dualización del sistema de protección social y se orienta a reforzar el polo asistencial de la protecció ${ }^{4}$ o, en términos de Netto (2012) la denominada "asistencialización minimalista de las políticas sociales".

Las organizaciones del campo asistencial configuran el espacio ocupacional privilegiado de las profesiones asistenciales. Estas profesiones se definen por el lugar ocupado en la división del trabajo y no por su naturaleza disciplinar. Las profesiones asistenciales son comprendidas como el conjunto de prácticas profesionales desarrolladas en la atención institucionalizada de las expresiones de la cuestión social que son objeto de políticas públicas, inscribiendo su espacio ocupacional dentro del sector servicios sociales, es decir, en aquellos servicios que se ocupan de responder a demandas colectivas de reproducción social. El ámbito de la reproducción social es entendido "en el sentido amplio de la recreación del reconocimiento de las pautas básicas que orientan la vida social y de la consecuente continuidad elemental de las prácticas sociales; pero también en lo que atañe, específicamente, a la reproducción de la vida y de la fuerza de trabajo" (Grassi, 2003, p. 25).

Así entendidos, el campo asistencial y la práctica de las profesiones asistenciales se configuran como espacios contradictorios en el marco de la administración burguesa de las manifestaciones de la cuestión social y sus pautas de reproducción social.

\section{La institucionalización de las políticas de combate a la pobreza}

El recrudecimiento de la pobreza como consecuencia del ajuste estructural de la década del 90 fue uno de los principales argumentos utilizados para promover la creación de una nueva institucionalidad para su atención. Midaglia et al. (2010, p.

4. En el documento titulado Programas sociales y transferencias de ingresos en Uruguay: lo beneficios no contributivos y las alternativas para su extensión (Arim, Cruces, Vigorito, 2009), un grupo de investigadores del Instituto de Economía de la Universidad de la República que han asesorado en la construcción e implementación del Plan de Emergencia Social y del nuevo régimen de Asignaciones Familiares del Plan de Equidad, exploran las posibilidades de expandir las prestaciones no contributivas en Uruguay. 
126) señalan que los Ministerios Sociales instalados en varios países latinoamericanos desde mediados de los 90, se han especializado "en el tratamiento de problemáticas sociales en los que predominan criterios de insuficiencia socioeconómica y/o de atributos particulares de sectores poblacionales — grupos étnicos, de género y etarios, entre otros."

Esta especialización institucional, o si se quiere, el cometido de esta nueva institucionalidad será, entonces, la de garantizar la protección social de grupos poblacionales cuya vulnerabilidad reposa en su condición de pobreza o en su carácter de portadores de ciertos atributos que representan un handicap social. En otras palabras, los Ministerios Sociales son, por definición, las instituciones responsables de llevar adelante iniciativas protectoras dirigidas a los individuos pobres y/o minusválidos sociales en el sentido que le atribuye Castel (2003). La institucionalidad naciente también responde a la necesidad de gestionar una multiplicidad de iniciativas diversas y dispersas que focalizan sus prestaciones en estos grupos sociales. La asistencia social — o la "seguridad social" no contributiva de la pobreza - es el campo de competencia institucional de los nuevos Ministerios Sociales. Su creación parece anunciar que los programas de combate a la pobreza o de transferencias de renta llegaron para quedarse reconfigurando el campo asistencial.

En el caso uruguayo, el MIDES tiene un papel central en el desarrollo de programas de combate a la pobreza pero secundario y auxiliar en su componente monetario. La competencia institucional central del MIDES respecto a las AFAM-PE es de carácter auxiliar limitándose a la construcción de bases de datos para la identificación de beneficiarios y la verificación de contrapartidas. La administración de las AFAM es competencia del BPS que puede auxiliarse en las bases de datos y el apoyo en comprobaciones y visitas en el MIDES. Es el BPS quien tiene el cometido de verificar y controlar el cumplimiento de los requisitos de elegibilidad para ser beneficiario o administrador. Es también el BPS quien puede requerir de los Ministerios de Educación y Cultura y de Salud Pública la información necesaria para comprobar la asistencia a centros educativos y los controles médicos así como demandar el apoyo del MIDES para realizar comprobaciones e inspecciones para determinar la existencia de las condiciones de elegibilidad y mantenimiento de la prestación.

La creación de Ministerios Sociales puede interpretarse como expresión institucional del quiebre del sistema bipolar de protección social y como confirmación de la respuesta sociopolítica construida que privilegia el despliegue de prestaciones de carácter asistencial dirigidas a sectores que ven amenazada su reproducción 
biológica y/o social. En este sentido, Midaglia et al. (2010, p. 150) indican que la presencia de esta nueva institucionalidad

(...) pone de manifiesto que las problemáticas relacionadas con la pobreza y vulnerabilidad social tienden a perdurar, y en algún sentido, están 'separadas', o mejor dicho, carecen de soluciones asociadas al mercado de empleo. Los Planes y políticas sociales que manejan estas Secretarías dejan en evidencia que se requiere de medidas específicas para tratar las actuales cuestiones sociales, y al parecer, esas iniciativas son tan particulares que se torna imposible o inconveniente ubicarlas en la esfera de los Ministerios de Trabajo.

De este modo, el trabajo como soporte privilegiado de inscripción social (Castel, 1997) está siendo desechado como respuesta posible. Pareciera que las transformaciones en el mundo del trabajo son asumidas como un "dato" de la realidad que el sistema político debe considerar para atender a sus víctimas y no como un espacio de relaciones sociales susceptible de ser direccionado, orientado, en otras palabras, intervenido por parte del poder político como lo fuera en el apogeo de los Estados Sociales.

Los objetivos de las otrora políticas de integración social son reescritos por la externalidad ${ }^{5}$ del Estado respecto al libre juego del mercado: los "inempleables", los "inútiles para el mundo" (Castel, 1997) son orientados hacia espacios de protección regidos por mecanismos no mercantiles. Mientras las políticas de integración se proponían la "búsqueda de grandes equilibrios, de la homogeneización de la sociedad a partir del centro", las políticas de inserción se orientan a la construcción de espacios sociales para quienes demuestran su inutilidad social y "pueden entenderse como un conjunto de empresas de elevación del nivel para cerrar la distancia con una integración lograda" (Castel, 1997, p. 422). En este sentido, vale la pena recordar cómo Midaglia y Silveira (2011, p. 243) describen los cambios en las AFAM: aquellas prestaciones, nacidas para asegurar una sociedad "civilizada y garantista", pretendían contribuir al proyecto de una nación moderna, "con una mano de obra joven, educada y un mercado de empleo en expansión" mientras que las actuales se convirtieron en políticas de combate a la pobreza que "buscan incidir en las condiciones de vida de los estratos sociales desfavorecidos".

5. Externalidad que no es tal cuando se trata de garantizar jurídicamente los intercambios contractuales y que, contradictoriamente, es defendida cuando se trata de regular para proteger a las poblaciones que participan del mercado con su único capital: su fuerza de trabajo. 


\section{La reconfiguración tecnológica del campo asistencial}

(...) importa mencionar un tema que aparece como secundario desde el punto de vista institucional, sin embargo cuando se trata en detalle se evidencia su relevancia política: el referido a los sistemas de información social. Los cometidos de coordinación, articulación y ejecución de intervenciones sociales intersectoriales, requieren necesariamente información confiable sobre las condiciones de vida de la población, su localización territorial, así como de las prestaciones sociales que brinda el Estado y su ubicación institucional. Si bien esta actividad, de perfil predominantemente técnico, es estratégica en el funcionamiento de los estos Ministerios, y de cierta forma legitima su presencia en la esfera estatal, genera también la oportunidad de restringir el manejo político de los temas relativos a vulnerabilidad y transferir su la conducción a los cuerpos "tecnocráticos", garantizando así el carácter "residual” de estos temas sociales y del marco institucional que los alberga (Midaglia et al., 2010, p. 151).

Esta compartible preocupación indica uno de los ejes de transformación reciente del campo asistencial. La emergencia de sofisticados sistemas de información ha sido posible en este momento histórico de gran desarrollo tecnológico. Sin embargo, la tecnificación creciente de la asistencia no puede ser explicada solamente como consecuencia de los avances en las tecnologías de la información y la comunicación, ni como efecto del desarrollo de nuevos saberes especializados. Si bien estos procesos constituyen prerrequisitos funcionales que posibilitan la tecnificación de la gestión asistencial, ésta también demanda formas de legitimidad que devienen del relativo consenso social acerca de los problemas que nos aquejan y las respuestas socialmente construidas.

En ese sentido, la insistente referencia a la falta de recursos públicos para financiar los requerimientos de protección social mantiene la misma fuerza que en la década del 90 pese al crecimiento económico registrado en varios países del continente. Con este argumento también se mantiene la recurrencia a la focalización como instrumento para orientar la protección hacia los grupos más vulnerables en un contexto de "escasos" recursos. De este modo, se fundamenta la focalización de las prestaciones del nuevo régimen de asignaciones familiares del Plan de Equidad:

Sin desconocer los problemas asociados con la focalización, y en función de una restricción presupuestal que se tradujo explicitamente en una población objetivo definida en términos cuantitativos, en el caso de las nuevas Asignaciones Familiares 
se optó por utilizar un mecanismo de identificación de beneficiarios que permitiera una mejor aproximación a los niños y adolescentes con mayor vulnerabilidad socioeconómica (Amarante et al., 2008, p. 4). ${ }^{6}$

Despojado de sus aristas político-ideológicas el problema se reduce a su dimensión técnica: ¿cómo construir un instrumento "justo" para identificar a los "más vulnerables" cuando el "presupuesto" define a priori el número posible de beneficiarios? o ¿cómo identificar a los pobres merecedores de ayuda?

Si bien estas interrogantes recorren la historia del campo asistencial son otros los agentes profesionales, los saberes disciplinares y las tecnologías a las que se apela para darles respuesta en la contemporaneidad. La creciente tecnificación del campo asistencial con la consecuente demanda de conocimiento experto especializado ha producido transformaciones en la división del trabajo técnico-profesional. Es así que la identificación de los pobres merecedores de ayuda dejó de ser monopolio de la práctica profesional del Trabajo Social. La población receptora de las AFAM-PE es definida por su condición de vulnerabilidad la cual se determina según criterios estadísticos "teniéndose en cuenta, entre otros factores: ingresos del hogar, condiciones habitacionales y del entorno, composición del hogar, características de sus integrantes y situación sanitaria". 7 Se promueve el desarrollo de instrumentos "objetivos", "impersonales", "neutros", "insensibles” de selección de los más pobres entre los pobres. En el caso uruguayo, los responsables del diseño de un indicador de vulnerabilidad socioeconómica - denominado Índice de Carencias Críticas (ICC) - que permitió ordenar a los hogares postulantes al nuevo sistema fueron economistas de la Universidad de la República.

Sin embargo, contrariando el espíritu de sus creadores, estos instrumentos deben menos su eficiencia a su "naturaleza objetiva" - aunque sí logran definir un conjunto de variables de difícil manipulación por parte del postulante - que a la racionalidad gerencial que los orienta y que minimiza el recurso a la racionalidad clínica característica de las profesiones asistenciales puesta bajo sospecha por su "discrecionalidad subjetivista". La racionalidad clínica encuentra en la eficacia uno de sus principios estructurantes al tiempo que la eficiencia ocupa ese estatuto para

6. La Ley n. 18.227 de creación del nuevo régimen de asignaciones familiares estableció que niños y adolescentes pertenecientes al primer quintil de ingresos de la población del país recibirían la prestación en 2008 (330.000) y la totalidad de los niños y adolescentes en situación de pobreza en 2009 (500.000).

7. Ley n. 18.227. 
la racionalidad gerencial. La eficacia promueve la búsqueda del camino más apropiado para la resolución de un problema mientras que la eficiencia orienta la búsqueda hacia la economización de la respuesta a ese problema. El efecto esperado o deseado es orientador de la racionalidad clínica mientras que el énfasis en la capacidad de disponer recursos para conseguir ese efecto es propio de la racionalidad gerencial. Valga a título de ejemplo el informe de la Contaduría General de la República de Costa Rica respecto al programa "Avancemos" donde se registra la siguiente observación: el Sistema de Información de la Población Objetivo (SIPO) establece cuatro categorías de beneficiarios. La categoría 3 fue ampliada de forma de incluir "no sólo los pobres extremos sino también a las personas en condición de vulnerabilidad y riesgo social". Como consecuencia se abrió la posibilidad de la recepción del beneficio "si existe un riesgo potencial para la salud, vida, integridad o amenaza a los derechos de educación del niño o la niña; con la cual, se deja a discreción del trabajador social el otorgar el beneficio, dependiendo de cual sea su forma de ponderar las diferentes variables y su criterio particular". Producto de esta apertura aumentó la participación del grupo 3 cuya proporción pasó del 5,6\% del total de beneficiarios en diciembre de 2007 al 24,5\%. El informe recuerda que la Ley de creación del Instituto Mixto de Ayuda Social (IMAS) enfatiza la atención de la población en condición de pobreza extrema siendo prioritaria la cobertura de los grupos 1 y 2 y alerta sobre el crecimiento de beneficiarios del grupo 3 aún cuando la cobertura en las dos primeras categorías apenas alcanza al 33 y $37 \%$ respectivamente.

Como fuera mencionado, en Uruguay se construyó el Índice de Carencias Críticas (ICC) para seleccionar a los beneficiarios de las AFAM-PE y controlar este tipo de situaciones "no deseadas". En la evaluación de la Tarjeta Uruguay Social (TUS) se destaca que el objetivo del ICC es traducir la situación socioeconómica de cada núcleo familiar a una expresión numérica dejando "de lado las discrecionalidades que puede tener un abordaje netamente cualitativo". Así mismo, en dicho informe se valora la eficiencia del uso del ICC pues "permite realizar un abordaje a una cantidad muy grande de casos" (TUS, 2011, p. 10).

En definitiva, si bien es cierto que el desarrollo tecnológico hizo funcionalmente posible este tipo de alteraciones en el campo asistencial, ha sido el "triunfo cultural del neoliberalismo" (Grassi, 2003) lo que las promovió y legitimó jerarquizando valores tales como la eficiencia y la eficacia frente a la solidaridad o la igualdad. Los imperativos de una gestión eficiente impulsan la reconfiguración tecnológica del campo asistencial y atraviesan la práctica de sus agentes (ya sean 
profesionales o no) ya sea cambiando su naturaleza, reforzando la división sociotécnica del trabajo, estandarizando procedimientos etc.

Por otro lado, la incorporación de tecnología está reconfigurando el campo asistencial mediante la economización de sus dispositivos. Los PTRC alcanzan importantes niveles de cobertura (focalizada) sin representar, necesariamente, una "ampliación del aparato estatal" pues, tal como afirman Midaglia y Silveira (2011, p. 227) "transfieren directamente renta a los beneficiarios sin intermediarios". De este modo, el dispositivo gana en eficiencia al sustituir a los funcionarios y profesionales intermediarios de la asistencia por conocimiento experto condensado en instrumentos de tecnificación que, una vez construidos, se autonomizan espacial y temporalmente de su creador. En realidad, si existiera voluntad política de mejorar las prestaciones y no sólo la cobertura de los servicios de educación y salud, el aparato estatal se ampliaría por efecto de la exigencia de contraprestaciones. Si el aparato estatal no se amplía como consecuencia del reforzamiento de los sistemas de salud y educación es debido a la despreocupación liberal por la calidad de los servicios públicos y la preocupación, también liberal, de promover su mercantilización para los sectores no pobres de población. En otras palabras, la precariedad de los servicios públicos se convierte en herramienta para promover su compra en el mercado por parte de sectores de ingresos medios y altos.

Las más recientes evaluaciones de impacto de los principales PTRC implementados en América Latina indican que se ha mejorado el acceso de sus beneficiarios a los servicios de salud y educación al tiempo que "no hay información concluyente en relación con objetivos finales de desarrollo humano, como el aprendizaje (Reimers, DeShano da Silva y Trevino, 2006) y el estado de salud o nutricional de niños y niñas" (Cecchini y Madariaga, 2011, p. 119). Parece comprensible un resultado así cuando se aumenta la cobertura incorporando a la población con mayores déficits y hándicaps en salud y educación sin incrementar las capacidades estatales de forma coherente con la magnitud de los problemas a enfrentar.

Tal vez, los débiles controles sobre el cumplimiento de las condicionalidades respondan a las dificultades de los sistemas educativo y sanitario para dar respuesta a las demandas esperables ante un férreo seguimiento de las mismas. La extensión de los servicios de salud y educación es un prerrequisito indispensable para exigir el cumplimiento efectivo de las condicionalidades. 
En pocas palabras, las transformaciones señaladas no son, esencialmente, producto del desarrollo tecnológico sino de su particular uso motivado en la economización de los dispositivos de protección social y en la jerarquización de los principios de la racionalidad gerencial para la gestión de lo social.

\section{Nuevos procesos de división sociotécnica del trabajo profesional en el campo asistencial}

La emergencia de los PTRC supone una reconfiguración del campo asistencial que, pese a la sustitución creciente de trabajo vivo, no conduce necesariamente a una mínima expresión de las profesiones asistenciales. Si, como vimos, el aparato estatal fuera ampliado como resultado de una inversión coherente con la magnitud de los desafíos en los sistemas de salud y educación, muy probablemente, aumentaría el espacio ocupacional para los profesionales de la asistencia.

Así mismo, la literatura sobre los PTRC en América Latina indica que las transferencias suelen ir acompañadas de cuatro clases de servicios dirigidos a las familias y a las comunidades. Algunos de estos servicios son intermediados por agentes profesionales tradicionalmente vinculados al campo asistencial: i) inserción laboral y generación de ingresos; ii) orientaciones, charlas educativas y talleres temáticos; iii) "acompañamiento familiar" con el propósito de hacer un seguimiento de las condicionalidades y de proveer apoyo psicosocial; y iv) mejoramiento de la infraestructura (Cecchini y Madariaga, 2011). En especial, el componente denominado "acompañamiento familiar" es rescatado como una prestación crecientemente aceptada en la región siendo caracterizada como la "visita de profesionales a los hogares usuarios con distintos objetivos" identificando dos versiones de este componente: "una que tiene como finalidad el seguimiento de las condicionalidades y otra orientada a la superación de las barreras psicosociales y culturales para lograr una plena inclusión social de los destinatarios" (Cecchini y Madariaga, 2011, p. 77). En el primer caso, la acción profesional se limita a suministrar información sobre los programas y servicios disponibles y facilitan el acceso de los beneficiarios: "el profesional de acompañamiento se transforma en el nexo entre el programa y la familia y genera un flujo de información que permite retroalimentar al programa sobre los problemas que enfrentan las familias para cumplir con las condicionalidades" (Cecchini y Madariaga, 2011, p. 78). La segunda modalidad 
se centra en el abordaje de aquellos aspectos psicosociales vistos como obstáculos para el acceso de los beneficiarios al mercado laboral y a los servicios educativos y sanitarios: "El apoyo psicosocial busca abordar aspectos asociados a la integración y que se refieren a la dinámica familiar, como la autoestima, y representa una forma de acercar a los destinatarios a la oferta de servicios y programas sociales" (Cecchini y Madariaga, 2011, p. 78). En este caso, la experiencia de Chile Solidario parece que marca los debates. Los agentes profesionales acompañan a las familias beneficiarias por un período de dos años en el supuesto de que la incorporación de apoyos familiares es un elemento central para combatir la pobreza.

En el caso de las AFAM-PE también se prevén un conjunto de prestaciones no monetarias del tipo descripto con alguna participación de agentes profesionales tradicionalmente vinculados al campo asistencial. Sin embargo, en lo que refiere estrictamente a la transferencia monetaria se observa que:

i) el acceso a la prestación no supone la mediación de un agente asistencial sino una declaración jurada del postulante frente a un funcionario administrativo. En casos "dudosos" se recurre a la "entrevista" domiciliaria por parte de operadores mayoritariamente no profesionales aunque sí medianamente calificados;

ii) no se ha previsto un protocolo o itinerario de intervención profesional vinculado al (in)cumplimiento de las condicionalidades. El ítem sobre contrapartidas a exigir y mecanismos de control (Plan de Equidad, s/f, p. 34) establece que para efectivizar las condicionalidades "se conformará un sistema único de registros de Asignaciones Familiares además de consolidar proyectos en curso destinados a la creación de un sistema integral de información en el Estado (SIIAS)". Es decir, que los mecanismos de control y seguimiento se reducen a la construcción de sistemas de información desconsiderando, al menos en su elaboración inicial, otras modalidades de acompañamiento que supongan trabajo profesional en espacios de relación cara a cara.

La reconfiguración del campo asistencial a partir de la irrupción de los PTRC no permite concluir que las profesiones asistenciales serán prescindibles en un futuro más o menos próximo. Más bien se trata de una reconfiguración que conduce a una nueva división del trabajo profesional que responde a los requerimientos de la administración gerencial de la asistencia, con la cual la eficiencia se 
constituye en un vector central en la estructuración del campo. En la experiencia de Chile Solidario el "acompañamiento familiar" es un componente privilegiado. En el caso uruguayo si se extiende el análisis a la Red de Asistencia e Integración se observan líneas de trabajo que demandan la práctica de las profesiones asistenciales. A título de ejemplo, las acciones previstas para la atención de la primera infancia suponen la "captación temprana de las mujeres embarazadas", "visitas domiciliarias en las situaciones de riesgo", "incorporación de programas de estimulación" (Plan de Equidad, s/f, p. 39). De este modo, las alteraciones observadas parecen indicar una metamorfosis de la naturaleza del quehacer de las profesiones asistenciales y no su desaparición por su supuesta pérdida de utilidad social. ${ }^{8}$ Con la reconfiguración del campo asistencial se radicaliza la división del trabajo técnico y puede mutar la naturaleza del quehacer de las profesiones asistenciales. Sin embargo, la necesidad social de estas profesiones está fundada en los atributos característicos de la reconfiguración del campo asistencial: las profesiones asistenciales son demandadas para el registro de las particularidades individuales y la administración de las "fragilidades individuales" en espacios de relación "cara a cara" para luego diluir estas particularidades en un nuevo campo documental constituido por las cada vez más sofisticadas bases de datos y sistemas de información.

Las alteraciones tecnológicas e institucionales del campo asistencial complejizaron la división sociotécnica del trabajo. Agentes profesionales portadores de conocimiento experto cada vez más especializado comparten el mismo espacio ocupacional con agentes cuyas prácticas requieren mínimas capacidades y destrezas. Los primeros se ocupan de las tareas inherentes a la ingeniería política de los dispositivos mientras que los segundos llevan adelante las tareas de relacionamiento "cara a cara" con los usuarios de la asistencia. Como fue dicho, las profesiones asistenciales se enfrentan a una transformación de sus prácticas habituales que puede modificar su propia naturaleza. Estas innovaciones tecnológicas tienen, por

8. Mitjavila (2006) recupera diversos análisis (Haraway, 1991; Castel, 1981; Rabinow, 1991; Mitjavila, 1999) acerca del impacto de las nuevas formas de gestión social sobre las prácticas de asistencia directa. De ahí se desprende que para algunos investigadores estas prácticas tienden a desaparecer siendo sustituidas por redes de comunicación y gerenciamiento. Para otros, estos procesos son síntoma de una progresiva pérdida de poder de los agentes asistenciales frente a los administradores de la asistencia. Por último, otras lecturas interpretan que las nuevas tecnologías políticas de gestión requieren tanto el gerenciamiento político-administrativo de la población como la individualización de los riesgos como condición de posibilidad para la construcción del mapa. 
ejemplo, la capacidad de desplegar "espacios virtuales" de intervención sobre las fragilidades individuales que sustituyen la relación directa del profesional con el usuario. En la página web del Instituto Nacional de Alimentación se describe el uso dado a las computadoras que poseen los niños y adolescentes matriculados en la educación pública primaria y secundaria en Uruguay:

A través de la computadora que cada niño posee se podrán enviar:

- Mensajes generales informando acerca de pautas de alimentación saludables.

- Mensajes personalizados sobre consumos realizados y propuestas sobre cambios en las pautas de alimentación.

- Mensajes personalizados sobre aspectos vinculados a la salud del niño y su familia (vacunas, controles obstétricos etc.). ${ }^{9}$

De este modo, se configura un espacio virtual de administración de fragilidades individuales, impensable en el pasado reciente, que repercute fuertemente en la naturaleza de la práctica asistencial. ${ }^{10}$

Fenómenos similares derivados de los procesos de reestructuración productiva son observables ante la reconfiguración del campo asistencial y la mayor fragmentación entre sus operadores. ${ }^{11}$ Agentes profesionales portadores de conocimien-

9. Disponible en: $<$ http://www.inda.gub.uy $>$, $<\mathrm{http}: / /$ www.inda.gub.uy $>$. Acceso el: 12 dic. 2009.

10. Huws $(2009$, p. 49$)$ describe esta posibilidad del siguiente modo:

"As habilidades requeridas para operar um computador e seus numerosos acessórios de comunicação não devem ser, obviamente, confundidas com a totalidade dos requisitos de um dado emprego. Frequentemente, eles estão subordinados a outras habilidades 'centrais'-as aptidões requeridas para o emprego 'em si'. Entretanto, este também pode se submeter a um processo de modificação (que pode tomar a forma de rotinização ou total mercadorização) que está mudando a sua natureza. Trabalhadores sociais, por exemplo, podem ver-se preenchendo formulários padrões numa tela de computador em vez de redigi-los no papel ou entregar relatórios mais nuançados e profissionalmente qualificados para seus clientes; professores podem ver-se administrando testes padrões [...]. Frequentemente, essas transformações são disfarçadas por uma mudança na divisão do trabalho. A descrição do emprego de um profissional pode ser fatiada em seu núcleo e o número de pessoas reduzido, ao passo que os antigos componentes do emprego que são capazes de rotinização são transfiridos para trabalhadores de baixa qualificação. Assim, por exemplo, perguntas rotineiras feitas a um sistema de ajuda podem ser respondidas por meio do uso de respostas automáticas por e-mails ou por um pessoal Junior, ao passo que somente problemas realmente difíceis seriam encaminhados para experts melhor remunerados. Ou pessoas doentes poderiam ser encorajadas por enfermeiras a ligar para um call center antes de marcar uma consulta com um médico [...]".

11. En igual sentido, Raichelis (2010) indica que las "pesquisas junto às empresas e ao setor privado realizadas por Dal Rosso (2008) concluíram que as tecnologias da informação intensificam os processos de trabalho, produzem um efeito mais controlador sobre o trabalho, organizam e encadeiam as tarefas de modo 
to experto cada vez más especializado comparten el mismo espacio ocupacional con agentes cuyas prácticas requieren mínimas capacidades y destrezas. Los primeros se ocupan de las tareas inherentes a la ingeniería política de los dispositivos mientras que los segundos llevan adelante las tareas de relacionamiento "cara a cara" con los usuarios de la asistencia. Mientras la rutinización, estandarización, tercerización y precarización marcan las trayectorias de las mayorías descalificadas, en el otro extremo, el trabajo experto se enfrenta a exigencias de creatividad y creciente especialización, mejores niveles salariales y condiciones laborales.

Si la reconfiguración del campo asistencial puede mudar la naturaleza de ciertas prácticas profesionales, cabe preguntarse acerca de su posible impacto sobre una de sus dimensiones configurativas centrales: la distribución del poder categorizador de lo social entre diversos espacios profesionales. Si se reconoce que un software no es "nada más que puro conocimiento en forma codificada", ${ }^{12}$ es pertinente interrogarse cuáles son las fuentes y quiénes los portadores del conocimiento posteriormente codificado. Históricamente algunas profesiones reivindican para sí el poder categorizador sobre ciertas situaciones o comportamientos. La psiquiatría, la medicina y la psicología representan claros ejemplos donde se reconoce el monopolio profesional sobre ciertas categorías diagnósticas. No exento de luchas, estas profesiones encuentran un lugar más o menos delimitado dentro del campo de la salud. Sus agentes profesionales ocupan un lugar particular en la división social del trabajo a través del cual las clasificaciones que elaboran tienen efectos sobre las formas de comprender la salud y la enfermedad, los procesos diagnósticos, los tratamientos indicados etc.

Sucede del mismo modo con los espacios de intervención reivindicados por los distintos agentes (profesionales o no) que operan en el campo de la asistencia. Cuando los PTRC seleccionan sus beneficiarios mediante la construcción de "algoritmos" y controlan contrapartidas con el auxilio de sistemas de información ¿sobre qué saberes y prácticas profesionales se funda el poder categorizador de lo social?, ¿cuáles son los saberes y las prácticas profesionales privilegiadas cuando se trata de clasificar poblaciones merecedoras de ayuda? Indicios de una respuesta posible se encuentran en el siguiente fragmento del informe de evaluación de la Tarjeta Uruguay Social (2011, p. 10) que describe el índice de carencias críticas

que desapareçam os tempos mortos, quantificam as tarefas realizadas e permitem a avaliação fiscalizatória do desempenho, entre outros".

12. La expresión corresponde a Detlev Hoch y es extraída de Castillo (2009, p. 15). 
(ICC) — instrumento elaborado por economistas para la selección de beneficiarios de las AFAM-PE - como: "una combinación y ponderación de distintas características no monetarias de los hogares que dan cuenta de su grado de vulnerabilidad. El objetivo del ICC es traducir a una expresión numérica la situación socioeconómica de cada núcleo familiar, realizando una valoración integral de la misma”.

\section{La tecnificación como domesticación del factor humano en el campo asistencial. La minimización de lo aleatorio en nombre de la eficiencia}

La tecnificación e informatización del quehacer profesional en el campo asistencial es una característica de la administración gerencial de la asistencia y supone nuevas formas de gestionar y controlar el trabajo profesional. La estandarización de las prácticas es el mecanismo que permite la gestión y el control del trabajo profesional y la informatización es el instrumento tecnológico que la vuelve posible.

Sin embargo, el gobierno absoluto de lo aleatorio continuará siendo una ensoñación tecnocrática mientras no se pueda prescindir del trabajo humano. Valga, a título de ejemplo, la siguiente situación:

La sofisticación de los mecanismos de verificación y la calibración de los tiempos de operación pueden volverse completamente ineficaces cuando, en la práctica, los encargados de ejecutar dichas acciones atienden consideraciones de otro tipo. Se ha registrado que, dadas las deficiencias de la oferta de servicios públicos y las dificultades que pueden enfrentar las familias pobres para cumplir con las exigencias de condicionalidades, en algunos casos, los operadores locales tienden a reportar el cumplimiento de las condicionalidades aun cuando no lo haya, ya que quitar los beneficios a ciertas familias implica dejarlas sin una fuente importante de sustento (Villatoro, 2008; Cecchini y Madariaga, 2011, p. 93).

Es este tipo de afectación del factor humano el que se quiere minimizar con la introducción de instrumentos fuertemente tecnificados y formalizados. En la medida en que la prescindencia del factor humano es imposible, la estandarización de las prácticas del campo asistencial contribuye a minimizar los espacios de incertidumbre y aleatoriedad, característicos del comportamiento humano, sujetándolos al seguimiento y apego a reglas y procedimientos estandarizados. Los proce- 
dimientos instrumentalizados y estandarizados estrechan los límites para el ejercicio de una práctica profesional autónoma y, como vimos, pueden afectar su naturaleza.

La adopción de procedimientos estandarizados suele ser defendida como estrategia ante formas clientelares de acceder a las prestaciones sociales. ${ }^{13} \mathrm{Sin}$ embargo, las evaluaciones sobre los PTRC en América Latina suelen hacer referencia a denuncias en tal sentido que demostrarían que la corrupción y el clientelismo "transversalizan" a los instrumentos y colocan en jaque su "naturaleza" o "esencia" objetiva.

Estas formas de controlar la incertidumbre también contribuyen a transformar la naturaleza del quehacer profesional en el campo asistencial. La economización de los dispositivos de vigilancia se realiza a través de la sustitución de trabajo vivo por tecnologías que permiten vigilar sin confinar. De este modo, la vigilancia poblacional se reorganiza sobre nuevas bases inaugurando formas de registro documental y habilitando formas innovadoras de tratamiento de la información.

Con la introducción de las tecnologías de la información y la comunicación para economizar los dispositivos de vigilancia se altera el carácter de este tipo de práctica: al igual que en el panóptico de Bentham analizado por Foucault, quien vigila no es visto, pero ahora esta característica se vuelve radical pues no exige el confinamiento de los vigilados (Vecinday, 2011) o, en palabras de Bauman (2001, p. 47), las nuevas técnicas de poder desvinculan a "controladores" de "controlados". Las TICs son tecnologías tanto de producción como de consumo de información (Huws, 2009, p. 38) y esta es su principal virtud para el desarrollo de funciones de vigilancia poblacional. En el caso de las AFAM-PE, la vigilancia poblacional se sirve del uso del Plan Ceibal ${ }^{14}$ y la tarjeta alimentaria (Tarjeta Uruguay Social — TUS).

El Plan Ceibal supone la identificación de cada niño a través de la computadora que le es asignada. De este modo, las computadoras del Plan Ceibal están siendo utilizadas para vigilar el cumplimiento de las contrapartidas en salud y educación:

13. El informe de evaluación de la Tarjeta Uruguay Social destaca la virtuosidad del uso del índice de carencias críticas (ICC) pues "proporciona transparencia al proceso" (TUS, 2011, p. 10).

14. El Plan Ceibal (Conectividad Educativa de Informática Básica para el Aprendizaje en Línea) se trata de un programa educativo desarrollado en el ámbito de la educación primaria a nivel público a través del cual cada niño y cada docente recibe un computador para uso personal. 
[...] el Plan Ceibal está sirviendo para el contralor de la asistencia escolar. Entonces se ha agregado una nueva posibilidad, no solamente la asistencia, el pasado de lista en la escuela, sino también de esta forma. Y con la salud también estamos haciéndolo a partir de los cruces de información con el nuevo Sistema Nacional Integrado de Salud. ${ }^{15}$

Por otra parte, pero en igual sentido, la TUS es valorada por su "alta trazabilidad", lo que significa que "se podrá conocer el detalle de los artículos comprados, limitar o anular determinados artículos y establecer montos máximos por tipo de alimentos" (Plan de Equidad, MIDES, s/f, p. 57) Esta particular cualidad tecnológica también es destacada en el informe elaborado por las instituciones administradoras de la TUS (2011, p. 13) pues se produce y se consume información para la toma de decisiones de gestión cuando "se cuenta con información estadística detallada sobre qué se adquiere, quién, dónde, cuándo, en qué cantidades, brindando información por zona, departamento etc., permitiendo así absoluta trazabilidad del consumo realizado por los titulares".

Y también se produce y consume información para la toma de decisiones "biográficas" y controlar contrapartidas cuando se envían "mensajes a los beneficiarios a través de los tickets de compra, dando lugar a un nuevo medio de comunicación".

\section{Consideraciones finales}

Junto a la emergencia y consolidación de los PTRC como herramienta principal de combate a la pobreza asistimos a la reconfiguración del campo asistencial tanto a nivel institucional como tecnológico. Estas alteraciones impactan directamente sobre las condiciones de trabajo y las prácticas profesionales de los agentes que operan dicho campo y también repercuten en los procesos de identificación y selección de los beneficiarios de las prestaciones asistenciales y en las formas de control y vigilancia sobre los comportamientos individuales.

Los PTRC son la expresión más acabada de la creciente institucionalización del proceso de individualización social a través del cual se responsabiliza al indi-

15. Entrevista al Sr. Ernesto Murro, Presidente del Directorio del Banco de Previsión Social, el 7/7/2009 en Radio Uruguay. 
viduo frente al manejo de su propia vida, y esta atribución de responsabilidad se deriva de la percepción y tratamiento de los problemas sociales como si fuesen problemas individuales.

Esta percepción es lo que justifica clasificar, contar y vigilar a los asistidos así como promover rutas de salida para retirar la "ayuda". Si bien estos rasgos son parte de la historia de la asistencia, es en la actualidad cuando el proceso de individualización social exige al individuo pagar los costos por los riesgos que asume al tiempo que se le "retiran" los soportes colectivos que, otrora, sirvieron de apoyo a la construcción de sí.

Si se considera que los gobiernos latinoamericanos destinan alrededor del 1\% de su PBI para estos programas parecen exagerados los recursos (materiales, cognitivos y tecnológicos) demandados para la administración gerencial - es decir, eficiente - de la asistencia. Sin embargo, estos recursos son imprescindibles para identificar a los más pobres y someterlos a un campo de visibilidad como pre-requisito de cualquier iniciativa de gobierno poblacional: ¿cuántos son?, ¿dónde están?, ¿quiénes son?, ¿qué hacen?, ¿cuál es el repertorio de trayectorias vitales del que disponen?, ¿cómo incidir sobre las trayectorias "no deseables"?. La "asistencialización minimalista de las políticas sociales" (Netto, 2012) desnuda la actual preocupación del gobierno poblacional: ya no se trata de integrar mediante el trabajo, tampoco se trata de mantener sano y educado a un ejército industrial de reserva sino que se trata de prevenir, controlar y vigilar aquellos comportamientos que, real o potencialmente, representan un problema de seguridad en un contexto de creciente desigualdad y fragmentación social.

En otro orden de asuntos, es fundamental para una agenda de investigación sobre este campo temático la realización de estudios empíricos para dar cuenta no sólo de los procesos de trabajo prescriptos idealmente sino de los procesos reales de trabajo profesional, es decir la organización y la actividad "ideal" y la organización y la actividad real, tal como lo sugiere Castillo (2009) para el estudio de los trabajadores del software refiriéndose al estudio de "situaciones reales de trabajo". En otras palabras, se trata de identificar las formas que efectivamente adoptan los esfuerzos por estandarizar los procedimientos profesionales en el campo asistencial junto con las respuestas de los diversos agentes profesionales.

Recebido em 11/2/2013 - Aprovado em 5/8/2013 


\section{Referencias bibliográficas}

ANTUNES, R. “O século XX e a era da degradação do trabalho.” En: SILVA, J. Pereira da (Org.). Por uma sociologia do século XX. São Paulo: Annablume, 2007.

BAUMAN, Z. La sociedad individualizada. Madrid: Cátedra, 2001.

CASTEL, R. Propiedad privada, propiedad social, propiedad de sí mismo: conversaciones sobre la construcción del individuo moderno. Rosario: Homo Sapiens, 2003.

. Las metamorfosis de la cuestión social: una crónica del salariado. Buenos Aires: Paidós, 1997.

CASTILLO, J. O trabalho do conhecimento na sociedade da informação: a análise dos programadores de software. En: ANTUNES, R.; BRAGA, R. (Orgs.). Infoproletários: degradação real do trabalho virtual. São Paulo: Boitempo, 2009. pp. 15-36.

CECCHINI, S.; MADARIAGA, A. Programas de Transferencias Condicionadas: balance de la experiencia reciente en América Latina y el Caribe. Cepal, 2011.

DOMÍNGUEZ UGÁ, V. A categoria "pobreza” nas formulações de política social do Banco Mundial. Rev. Sociol. Politica, n. 23, Curitiba, nov. 2004.

GRASSI, E. Políticas y problemas sociales en la sociedad neoliberal: la otra década infame (I). Buenos Aires: Espacio, 2003.

HUWS, U. A construção de um cibertariado? Trabalho virtual num mundo real. En: ANTUNES, R.; BRAGA, R. (Orgs.). Infoproletários: degradação real do trabalho virtual São Paulo: Boitempo, 2009. p. 37-58.

MIDAGLIA, C.; SILVEIRA, M. Políticas Sociales para enfrentar los desafíos de la cohesión social. Los nuevos programas de transferencias condicionadas de renta en Uruguay. En: BARBA, C.; COHEN, N. (Coords.). Perspectivas críticas sobre la cohesión social: desigualdad y tentativas fallidas de integración social en América Latina. Buenos Aires: Clacso/CROP, 2011. p. 225-250.

MIDAGLIA, C. et al. El significado político de los Ministerios Sociales en Argentina, Chile y Uruguay. Revista Chilena de Administración Pública, n. 15-16, p. 123-154, 2010.

MITJAVILA, M. El riesgo como instrumento de individualización social. En: BARKÚN, M.; KRMPOTIC, C. El Conflicto social y político: grados de libertad y sumisión en el escenario global y local. Buenos Aires: Prometeo Libros, 2006. p. 91-108.

MITJAVILA, M.; VECINDAY, L. El enfoque de riesgo como dispositivo individualizador en el campo social. En: LORENTE, B. (Org.). Transformaciones del Estado Social: pers- 
pectivas sobre la intervención social en Iberoamérica. Madrid/Buenos Aires: Miño y Dávila, 2011. p. 79-104.

NETTO, J. P. Crise do capital e consequências societárias. En: Serviço Social \& Sociedade, São Paulo, n. 111, 2012.

ORTEGA, E.; VECINDAY, L. De las estrategias preventivistas a la gestión del riesgo: notas sobre los procesos de individualización social. En: Revista Fronteras, Montevideo, Departamento de Trabajo Social, Facultad de Ciencias Sociales, UdelaR, p. 11-20, 2009.

RAICHELIS, R. Intervenção profissional do assistente social e as condições de trabalho no Suas. En: Serviço Social \& Sociedade, n. 104, p. 750-772, 2010. Disponible en: <http:// www.scielo.br/pdf/sssoc/n104/10.pdf>. Acceso el: 10 oct. 2012.

ROSANVALLON, P. La nueva cuestión social: repensar el Estado providencia. Buenos Aires: Manantial, 1995.

SILVA, Maria Ozanira da S. e. Pobreza, desigualdade e políticas públicas: caracterizando e problematizando a realidade brasileira. En: Katálysis, v. 13, n. 2, p. 155-163, 2010.

TENTI, E. Notas sobre exclusión social y acción colectiva: reflexiones desde Argentina. En: LAGUADO D., A. (Ed.). La política social desde la constitución de 1991: ¿una década perdida? Bogotá: Universidad Nacional de Colombia, 2000.

VECINDAY, L. Nuevas formas de vigilancia poblacional: el papel de los sistemas de información. En: CARVALHO JÚNIOR, Cesar Vaz de; FIGUEIRÔA, Edmundo de Sá; SENRA, Nelson de Castro; GONZÁLEZ BOLLO, Hernán (Orgs.). Em Associação das Américas, as estatísticas públicas como objeto de estudo. Publicações SEI, Salvador, p. 347-360, 2011. Disponible en: <www.sei.ba.gov.br>.

\section{Fuentes documentales}

AMARANTE, V. et al. Metodología para la selección de los beneficiarios del nuevo sistema de Asignaciones Familiares. Informe Final, 2008.

ARIM, R. et al. Programas sociales y transferencias de ingresos en Uruguay: los beneficios no contributivos y las alternativas para su extensión. Cepal, 2011.

INFORME TARJETA URUGUAY SOCIAL. Mides, 2012. Disponible en: <http:/www. mides.gub.uy/innovaportal/file/14984/1/informe_tus_2011_componente_alimentario.pdf $>$. Aceso en: 20 ago. 2012. 
Informe sobre el diseño y ejecución del programa de transferencia monetaria condicionada denominado Avancemos. Contaduría General de la República de Costa Rica, 2008. Disponible en: <http://www.asamblea.go.cr/Informes_de_la_Contraloria/Informes\%202008/informes_mayo_2008/DFOE-SOC-17-2008.pdf>. Aceso en: 20 ago. 2012.

Ley n. 18.227. Disponible en: <http://www0.parlamento.gub.uy/leyes/AccesoTextoLey. asp?Ley $=18227 \&$ Anchor $=>$. Aceso en: 12 jun. 2010.

Plan de Equidad, Ministerio de Desarrollo Social. Disponible en: <http://www.mides.gub. uy/innovaportal/file/913/1/plan_equidad_def.pdf $>$. Aceso en: 12 sept. 2012. 